\title{
Craving en adicciones conductuales: propuesta de un modelo teórico explicativo en la adicción a Facebook y mensajería instantánea
}

\section{Craving in behavioral addictions: proposal of an explicative theoretical model in addiction to Facebook and Instant messaging}

\author{
José Leiva-Gutiérrez \\ Escuela de Psicología, Programa de doctorado en psicología, Universidad Católica del Norte,Chile \\ Alfonso Urzúa M. \\ Escuela de Psicología, Universidad Católica del Norte, Chile \\ Recibido (25/05/2017) Aceptado (25/08/2017)
}

\begin{abstract}
Resumen
El craving se define como el deseo o ansias por introducir una sustancia en el cuerpo. La American Psychiatric Association ha propuesto que para adicciones conductuales, que implican el realizar algún tipo de acción, se consideren los mismos criterios que para una adicción a sustancias. Las naturalezas de los objetos son diferentes por lo que surge el desafío de analizar si el abordaje de las adicciones conductuales debe realizarse bajo los criterios de una adicción a sustancias. Se seleccionaron 23 artículos sobre la materia los que permitieron elaborar un modelo teórico explicativo de craving hacia Facebook y Mensajería instantánea. El modelo consta de tres dimensiones: intensidad del deseo, grado de control y expectativas de calma. Se propone estudiar la forma en que estas dimensiones se presentan en adicciones conductuales para reconstruir el concepto en este campo, y generar un instrumento que permita su evaluación.
\end{abstract}

Palabras Clave: Craving, smartphones, redes sociales virtuales.

\begin{abstract}
Craving is defined as the desire to introduce a substance in the body. The American Psychiatric Association proposed to research if behavioral addictions can be assessed with the substance addictions criteria. Nature of both objects are different so the challenge of analyze if assessment of behavioral addictions can be done with the same criteria of substance addictions. 23 articles about substance addictions were selected to elaborate a theoretical explicative model of craving to start research craving to Facebook and Instant Messaging. The model consists of three dimensions: desire intensity, degree of control and calm expectations. It is proposed to study how these dimensions are in behavioral addictions to rebuild the concept in this area, and generate an instrument to asses it.

KeyWords: Craving, smartphones, virtual social networks
\end{abstract}

Correspondencia: José Leiva Gutiérrez, Escuela de psicología, Universidad Católica del Norte Avenida Angamos \#0610, Antofagasta, Chile, Email: jlg007@ucn.cl 


\section{Craving y su rol en las adicciones a sustancias}

El craving se ha definido a lo largo de la historia como el deseo $\mathrm{o}$ ansias por introducir una sustancia en el cuerpo (Skinner y Aubin, 2010). Es un estado subjetivo, motivacional y emocional (APA, 2013; Tiffany, 1990) que se presenta cuando no se tiene la sustancia disponible o se está manteniendo la abstinencia (Chesa et al, 2004). Pese a que existe relativo acuerdo en que se trata de un deseo, a la hora de medirlo no es clara su definición, existiendo actualmente múltiples debates en torno a su conceptualización y naturaleza como concepto (i.e. Jáuregui, Bolaños, Carbonero y Valero, 2010; Jáuregui, Bolaños, Valero y Ruiz, 2012; Martínez, Graña y Trujillo, 2010; Pérez, Quiroga y Pérez, 2014; Zeni y Araujo, 2009). Sumado a esto, y pese a que es muy relevante en la dependencia de sustancias en general, la discusión respecto de su constitución y dimensiones ha durado más de un siglo (Araujo et al, 2015).

Se han propuesto distintos modelos que explican el rol que el craving posee en la teoría de las adicciones (Skinner y Aubin, 2010). Considerando el papel que juega en la mantención y constitución de las adicciones, estos modelos pueden agruparse en dos grandes líneas de desarrollo: mientras unos lo sitúan como un elemento esencial y determinante de la recaída (Drummond, 2001), otros lo consideran como insuficiente para explicar esta o la adicción misma por sí sola.

En la primera línea, se puede mencionar que hay dos grandes teorías tradicionales explicativas. La primera comprende el craving desde el condicionamiento clásico, y la segunda desde mecanismos cognitivos. Existe una tercera propuesta relacionada con modelos neuroadaptativos, que vendría a integrar los dos modelos anteriores (Chesa et al, 2004).

En la segunda línea, Tiffany (1990) propuso un modelo cognitivo que explicaría la relación entre el craving y la búsqueda de la sustancia. Según este modelo, el craving es controlado por procesos mentales automatizados mientras que el consumo mismo no. Esto debido a que no en toda recaída había craving previamente, o no todo craving conllevaba a una recaída. Dicho proceso podría estar siendo mediado por la disponibilidad de la sustancia que se desea. Sin embargo, existen pacientes que han reportado que el craving lo viven como un deseo muy intenso y que incluso lo mencionan como causa de sus recaídas (Merikle 1999).

\section{Craving en adicciones conductuales y nuevas tecnologías}

$\mathrm{Al}$ estudiar las adicciones conductuales, o no relacionadas a sustancias, se ha debatido cual es el lugar que ocupan en la teoría de las adicciones e incluso si pueden considerarse o no como adicciones propiamente tal (Martin y Petry, 2005). Se propone que es posible considerarla adicción debido a que existe una activación del sistema nervioso autónomo similar en ambos casos, y que existen variables relacionadas que se presentan de modo similar. Por ejemplo, se ha encontrado que tanto adictos al alcohol como jugadores patológicos poseen similares dificultades en el control de impulsos para tomar decisiones (Lawrence et al, 2009). Paralelamente, se contrapone a lo anterior el hecho que la adicción en su concepción más tradicional, resulta del impacto de una sustancia sobre los químicos del cerebro, y de los cambios en la estructura del mismo. Las adicciones a sustancias no poseen dicho efecto, sino que un efecto a largo plazo que puede ser adquirido y aprendido.

Dentro de las adicciones conductuales, la que se ha estudiado en mayor profundidad es el juego patológico, existiendo incluso una escala diseñada para evaluar craving en dicho contexto (Lourido, 2015). La adicción a uso de internet (Blaszczynski, 2006; Widyanto y Griffiths, 2006) y al uso de teléfonos smartphones (Chóliz, 2010) han sido propuestas desde hace algunos años como tópicos que debiesen ser tomados en consideración en investigaciones futuras. Esto dado su reciente aparición y consecuencias negativas para las personas que las padezcan.

En el DSM-IV, las adicciones a sustancias y al juego patológico aparecen juntas pero clasificadas como un desorden de uso de sustancias y un desorden de control de impulsos respectivamente (APA, 2000). Lo mismo ocurre en el DSM-V (APA, 2013), en dónde se ha propuesto que para investigar adicciones conductuales se especifique en qué medida es posible evaluarlas con los mismos criterios a adicciones conductuales. En este sentido, se debería profundizar en los mecanismos subyacentes, curso y síntomas para determinar si son paralelos o pueden evaluarse de la misma manera (Hormes, Kearns y Timko, 2014). Esto puede extrapolarse a revisar la cuenta de Facebook o servicios de mensajería instantánea en el teléfono móvil como whatsapp. Es posible mencionar aquí la aparición de instrumentos como el Cuestionario de Experiencias Relacionadas con Internet, CERI. Este cuestionario estuvo basado en los trabajos de De García et al (2002) y tiene como fundamento los criterios diagnósticos del DSM-IV para abuso de sustancias y juego patológico (Torrente, Piqueras, Orgilés y Espada, 2014).

Estudios recientes sobre adicciones conductuales, específicamente al uso de Facebook, han orientado la investigación hacia la identificación de patologías asociadas con el uso patológico de internet o tecnologías como las antes mencionadas, evaluando la adicción con los criterios 
propuestos por la APA (i.e Beranuy, Fernández,-Montalvo, Carbonell y Cova, 2016; Hormes et al, 2014; Elhai, Dvorak, Levine y Hall, 2017).

Surge la pregunta de si es posible evaluar craving en adicciones conductuales con los mismos criterios de las adicciones a sustancias. Para intentar dar respuesta a esta pregunta, creemos necesario en primer lugar una revisión del concepto a fin de identificar los elementos esenciales del mismo, sin perder el o los elementos nucleares que lo constituyen. Se realizó una revisión teórica para lo cual se recurrió a los buscadores Web of Science, Ebsco, Scielo y Scopus utilizando las palabras clave "craving", "sustancias", "drogas", y "dependencia". Se obtuvieron un total de 58 documentos en español e inglés que no tuviesen más de 15 años de antigüedad. Para seleccionarlos se realizó una lectura completa y se consideró a aquellos que evaluasen una adicción a sustancias de manera empírica con una escala validada al menos de contenido y de no más de 15 años de antigüedad y que abordaran población general o clínica y que consideraran adicción a drogas duras y alcohol. No se incluyó artículos que directamente abordasen adicciones a internet, Facebook o tecnologías afines (por ejemplo los que usaron el instrumento CERI) debido a que estos han sido creados basados en los criterios diagnósticos del DSM-IV para sustancias, lo cual viene a representar el problema que se trata en la presente revisión. Se seleccionaron un total de 23 artículos que fueron clasificadas en un fichero bibliográfico según si craving se consideró como unidimensional o multidimensional, las dimensiones que consideraron y la sustancia que consumían los participantes.

\section{Mediciones del concepto de craving en adicciones a sustancias}

Al revisar ejemplos de mediciones unifactoriales de craving (Tabla 1) es posible observar una tendencia a considerar esta variable como un deseo de consumir, lo que es concordante con la teoría propuesta anteriormente.

Tabla 1. Estudios que han evaluado craving de modo unidimensional

\begin{tabular}{|c|c|c|c|}
\hline Autores & Año & Adicción a & Dimensión \\
\hline López y Becoña & 2006 & Cocaína & $\begin{array}{l}\text { Craving global, ítems abordan: intensidad del craving en el momento } \\
\text { actual y semana anterior, frecuencia del craving durante la semana anterior, } \\
\text { craving ante estímulos ambientales relacionados, probabilidad de consumo } \\
\text { en una situación e disponibilidad de la sustancia, intención de consumo, } \\
\text { pérdida de control al empezar a consumir, pérdida de control al tener la } \\
\text { sustancia delante, anticipación de mejora de la abstinencia con el consumo } \\
\text { y anticipación de efectos positivos }\end{array}$ \\
\hline Zeni y Araujo & 2009 & Cocaína & Deseo de consumir \\
\hline $\begin{array}{l}\text { Martínez, Graña y } \\
\text { Trujillo }\end{array}$ & 2010 & Alcohol & Deseo de beber \\
\hline Jáuregui et al & 2010 & Comida & Deseo de consumir (se considera sólo esta escala) \\
\hline $\begin{array}{l}\text { Martínez, Graña y } \\
\text { Trujillo }\end{array}$ & 2011 & Alcohol & Deseo de beber \\
\hline Martíneza y Verdejo & 2011 & Distintas drogas & Craving global, ítems abordan: intensidad, frecuencia, circunstancias \\
\hline $\begin{array}{l}\text { Dido, Ledur, Amaral y } \\
\text { Araújo }\end{array}$ & 2011 & Pasta Base & Tiempo de abstinencia \\
\hline Jáuregui et al & 2012 & Comida & Deseo de comer \\
\hline $\begin{array}{l}\text { Calvo, Betancort y } \\
\text { Díaz }\end{array}$ & 2012 & Nicotina & Deseo de consumir \\
\hline $\begin{array}{l}\text { Pérez, Quiroga y } \\
\text { Pérez }\end{array}$ & 2014 & Distintas drogas & Deseo de consumir \\
\hline
\end{tabular}




\begin{tabular}{lccc}
\hline Autores & Año & Adicción a & Dimensión \\
\hline Piñeiro et al & 2014 & Nicotina & Dependencia \\
Gutiérrez et al & 2015 & Alcohol & Se toma como obsesión mental por beber \\
$\begin{array}{l}\text { Foster, Ecker, } \\
\text { Zvolensky y Buckner }\end{array}$ & 2015 & Marihuana & Deseo de consumir \\
$\begin{array}{l}\text { Magee, Lewis y } \\
\text { Winhusen }\end{array}$ & 2016 & Nicotina, cocaína & Deseo de consumir \\
\hline
\end{tabular}

Si bien hay escalas que consideran de manera unifactorial el craving, en ocasiones cada ítem evalúa una de las distintas dimensiones principales subyacentes al concepto, muchas de las cuales igualmente concuerdan con las dimensiones de instrumentos que abordan de manera multidimensional este constructo (Tabla 2).

Tabla 2. Estudios que han evaluado el craving de manera multidimensional

\begin{tabular}{|c|c|c|c|}
\hline Artículo & Año & Adicción a & Dimensiones del craving \\
\hline Guardia et al & 2006 & Alcohol & Deseo de beber, Desinhibición conductual \\
\hline $\begin{array}{l}\text { Montes, Urosa, Rubio, } \\
\text { Poyo }\end{array}$ & 2006 & Alcohol & $\begin{array}{l}\text { Tipo de deseo (craving/priming), Intensidad del deseo, Refuerzo, Grado } \\
\text { de control, Interferencia }\end{array}$ \\
\hline Pedroso, Castro y Araujo & 2009 & Marihuana & $\begin{array}{l}\text { Compulsividad (Falta de control), emocionalidad, expectativas, } \\
\text { intencionalidad (Intento de facto de usar la sustancia). }\end{array}$ \\
\hline $\begin{array}{l}\text { Araujo, Oliveira, Pedroso } \\
\text { y Castro }\end{array}$ & 2009 & Nicotina & $\begin{array}{l}\text { Se estudia de ambas formas, puntaje total y factores. } \\
\text { Unidimensional: Puntaje total de la escala, craving se asume como } \\
\text { deseo de consumir } \\
\text { Factor 1: Deseo, alivio de síntomas de abstinencia, intención de fumar } \\
\text { Factor 2: Anticipación de efecto positivo, Deseo de fumar, intención de } \\
\text { fumar (se reporta que cargan más en factor 1). }\end{array}$ \\
\hline Zeni y Araujo & 2011 & $\begin{array}{l}\text { Tabaco, Pasta } \\
\text { base }\end{array}$ & $\begin{array}{l}\text { A cocaína: una dimensión que evalúa distintos aspectos del craving } \\
\text { A Tabaco: refuerzo positivo y negativo }\end{array}$ \\
\hline Araujo et al & 2011 & Pasta base & Nivel de craving/Intensidad del mismo, falta de control \\
\hline Marín et al & 2011 & Cocaína & Intención de uso, Deseo de consumo, expectativas de consumo \\
\hline $\begin{array}{l}\text { Villalobos, Marín, } \\
\text { Templos y Rosendo }\end{array}$ & 2012 & Nicotina & $\begin{array}{l}\text { Anticipación de consecuencias positivas de fumar, Deseo de fumar, } \\
\text { Anticipación de alivio de retirada. }\end{array}$ \\
\hline Ismael y Baltieri & 2014 & Cocaína & $\begin{array}{c}\text { Intensidad, Frecuencia, Duración del craving, cambios en relación con } \\
\text { semanas anteriores y respuesta a medicación. Sólo se consideran } \\
\text { Intensidad, frecuencia y duración. }\end{array}$ \\
\hline Araujo et al & 2015 & $\begin{array}{l}\text { Tabaco - Mar- } \\
\text { ihuana - Pasta } \\
\text { base }\end{array}$ & $\begin{array}{c}\text { Cocaína: Intensidad, pérdida de control } \\
\text { Tabaco: refuerzo positivo y negativo } \\
\text { Marihuana: Emociones, Intencionalidad, Compulsividad (descontrol). }\end{array}$ \\
\hline
\end{tabular}


Al sistematizar las distintas formas de medir el craving es posible apreciar que hay dimensiones que son comunes en los estudios.

Es posible apreciar que existe un elemento que podría tomarse como transversal en la investigación que reduce el craving a su definición más simple, y es el que existe un deseo que puede tener un cierto nivel de intensidad. Al evaluar sólo la intensidad, se asume que la persona tiene un nivel de deseo aunque éste sea muy bajo. Así, un elemento central a considerar es que la persona tiene un deseo de usar estos servicios.

Por lo anterior, la primera dimensión identificada es la intensidad del deseo. Aparece'como la más medida y se refiere específicamente a la percepción de qué tan fuerte es el deseo de consumir la sustancia. Así, se considera entonces la valoración que la persona hace de la magnitud del deseo que siente cuando no ha revisado el teléfono durante un tiempo largo. Esta valoración puede estar situada entre poco intenso y muy intenso.

Igualmente, es posible identificar una segunda variable y es el grado de control que se tiene sobre el deseo. En este sentido, las escalas abordan en qué medida la persona pierde el control o, de manera positiva, en qué medida se puede reprimir el deseo de consumir. Aquí igualmente es posible incluir la dimensión mencionada en algunos estudios que es la desinhibición conductual, que hace alusión a la incapacidad de resistirse a consumir (Guardia et al, 2011). Se considerará como grado de control a la medida en que la persona sea capaz o no de resistir el impulso de ejecutar la conducta de revisar las redes sociales virtuales o el smartphone. Si bien en un estudio fue considerado específicamente como compulsividad (Pedroso et al, 2009), se propone evaluar desde la perspectiva de cuál es el nivel de control que tiene la persona. Dado que la conducta no posee un impacto biológico cuya privación genere estados de angustia como en el caso de otras drogas (Martin y Petry, 2005), es que la persona si bien puede realizar la conducta de revisar las redes sociales virtuales de manera impulsiva, es posible tener un grado de control sobre ese impulso.

En tercer lugar, diversos instrumentos evalúan una dimensión cognitiva que alude a expectativas que se tienen con el consumo. Estas expectativas aluden a que la sustancia calmará los síntomas de la abstinencia. En uno de los instrumentos (Zeni y Araujo, 2011), se hace la diferencia entre refuerzos positivos y negativos, que serían la tranquilidad y la baja de los síntomas de la abstinencia respectivamente. Se puede considerar que las expectativas se refieren al grado en que la persona considera que el revisar el teléfono o las redes sociales a través de él hará que se reduzca la ansiedad por no haber revisado el teléfono en un periodo prolongado de tiempo. En este plano, Beck, Newman y Wright (2000) proponen que parte de la búsqueda de la sustancia se relaciona con paliar los efectos negativos de la abstinencia. Por esto, se debe hacer la precisión que la calma que se busca, por ejemplo, al revisar las redes sociales virtuales no es necesariamente la misma que se busca ante la abstinencia de una droga dura, puesto que esta última genera efectos diferentes a nivel cerebral (Martin y Petry, 2005). Por esto, esta dimensión, es una en la que debe explorarse qué naturaleza posee en el campo de las adicciones conductuales en un estudio posterior.

Un elemento que puede ser considerado y que ha surgido de las críticas al concepto y al rol que se le atribuye, hechas por Tiffany (1990) es la búsqueda de la sustancia. Se coloca en la discusión puesto que si bien, no es una variable constitutiva, es un elemento que podría marcar una diferencia entre las adicciones a sustancias y conductuales, dado que las redes sociales y servicios de mensajería instantánea son más sencillos de acceder que sustancias ilícitas.

Así, para aplicar el concepto de craving a adicciones conductuales, se puede partir por considerar que existe un deseo de usar los servicios de mensajería instantánea y redes sociales virtuales, que puede tener una intensidad, la persona puede tener un nivel de control sobre él, y además implícitamente tener expectativas que una reducción de este deseo y al mismo tiempo de una calma del deseo. Estas dimensiones, serían las que dan forma al deseo que la persona tiene de usar los servicios mencionados. Si se asume que el craving es un deseo en sí mismo, la intensidad sería una dimensión, así como el grado de control sobre este y las expectativas que se posean del mismo.

\section{Conclusiones y futuras investigaciones}

Al momento de realizar una revisión teórica de todas las distintas maneras en que el craving ha sido evaluado, se pudieron identificar los componentes esenciales en todas las mediciones. Si bien, se ha definido como un deseo de consumir una sustancia, era necesario conocer si las distintas evaluaciones eran consistentes con esto.

Desde la revisión, es posible ver que si bien el concepto 
originalmente fue concebido para el deseo de sustancias que generan un efecto muy específico en el cuerpo, igualmente se ha propuesto el utilizarlo para adicciones conductuales. Esto lleva el estudio de las adicciones a un nuevo plano, en donde la persona es adicta a llevar a cabo una conducta y no a injerir una sustancia. Pese a las diferencias, existen elementos en común que mantienen estas últimas en el plano de las adicciones, y es el deseo de realizar la conducta, con todas las subvariables o dimensiones que esto implique. Pero el migrar el craving a un plano diferente implica que algunos elementos sean distintos, aporten en distinta medida, o sencillamente no existan, para dar paso a elementos distintivos de este nuevo contexto. Sin embargo, el elemento central del constructo, el deseo de consumir, es algo que no se modifica y que está igualmente presente.

Es importante hacer notar que si se toman las adicciones conductuales con los mismos parámetros de las adicciones a sustancias, es posible que entonces el concepto evalúe las primeras de manera forzada, o de un modo que quizás no esté abordando el constructo en la medida que corresponda. Por esto es relevante el rescate del elemento nuclear, a fin de que el proceso de aplicar la variable permita abordar el último contexto de un modo más congruente.

De generarse un instrumento que mida craving en adicciones a las tecnologías antes mencionadas, los datos cuantitativos obtenidos desde este instrumento deben sostener el uso de la teoría del craving en ese contexto (Popham, 1997). Aquí cabe preguntarse si el evaluar este constructo en adicciones conductuales pero con instrumentos diseñados para adicciones de otras naturalezas, generarían información que realmente esté reflejando el nivel de deseo o lo que se esté evaluando. Es por esto que en el proceso de identificación de variables que subyacen a un concepto, implica hacer en primer lugar una revisión de las dimensiones que se han estudiado previamente, a fin de identificar aquellas que podrían componerlo. De este modo se puede realizar una propuesta de modelo teórico que deberá ser explorado y profundizado en investigaciones futuras.

Se propone la realización de un estudio mixto con diseño exploratorio (Creswell, 2015), orientado a generar una escala para evaluar multidimensionalmente el craving, con entrevistas semiestructuradas siguiendo la estructura detectada y propuesta en el presente trabajo. Posteriormente se debe construir el instrumento con base esta información y estimar el ajuste de los datos a este modelo para, de este modo, darle una validez empírica al mismo.
Finalmente, hay distintas dificultades sociales y psicológicas relacionadas con adicciones a internet (Torrente et al, 2014) o a los teléfonos smartphones (Elhai et al, 2017). Esto hace que contar con un cuerpo teórico más apropiado para la medición de craving en adicciones conductuales hacia estas tecnologías cobre especial relevancia en el ámbito clínico. Así, se contará con información que permita intervenir el mismo de manera eficaz y adecuada.

\section{Referencias}

American Psychiatric Association. (2000). Diagnostic and Statistical Manual of Mental Disorders 4ta edición. Washington, DC: American Psychiatric Association.

American Psychiatric Association. (2013). Diagnostic and Statistical Manual of Mental Disorders, 5ta edición. Washington, DC: American Psychiatric Association.

Araujo, R., Castro, M., Siqueira, R., dos Santos, P., Leite, L., Rossoni, M., y Roselli, A. (2011). Validação psicométrica do Cocaine Craving Questionnaire-Brief - Versão Brasileira Adaptada para o Crack para dependentes hospitalizados. Journal Brasileiro de Psiquiatria, 60, $233-239$.

Araujo, R., Castro, M., Siqueira, R., Lucena, P., Dido, A., Jobim, V., y Roselli, A. (2015). Induction and comparison of craving for tobacco, marijuana and crack. Archives of Clinical Psychiatry, 42, 117 - 121.

Araujo, R., Oliveira, M., Pedroso, R., y Castro, M. (2009). Coping strategies for craving management in nicotine dependent patients. Revista Brasileira do Psiquiatria, 31, 89 - 94.

Beck, A., Newman, C., y Wright, F. (2000). Terapia cognitiva de las drogodependencias. Barcelona: Ediciones Paidos Ibérica.

Beranuy, M., Fernandez-Montalvo, J., Carbonell, X., y Cova, F. (2016). Características del uso de Internet en los cibercafés. Terapia psicológica, $34,5-14$.

Blaszczynski, A. (2006). Internet Use: In search for an Addiction. International Journal of Mental Health and Addiction, 4, 7 -9.

Calvo, F., Betancort, E., y Díaz, M. (2012). Validation of the BASR relaxation technique for the control of craving, anxiety and stress in an experimental nicotine withdrawal set. Revista Latinoamericana de psicología, 44, 163 - 173.

Chesa, D., Elías, M., Fernandez, E., Izquierdo, E., y Sitjas, M. (2004). El craving, un componente esencial de la abstinencia. Revista Española de Neuripsiquiatría, 89, $93-112$.

Choliz, M. (2010). Mobile phone addiction: a point of issue. Addiction, $105,373-375$.

Creswell, J. (2015). A concise introduction to mixed methods research. Washington, DC: SAGE. 
De García, M., Vigo, M., Fernández, M., y Marcó, M. (2002) Problemas conductuales relacionados con el uso e Internet: Un estudio exploratorio. Anales de Psicología, 18, 273 - 292.

Dido, A., Ledur, G., Ferreira, A., y Araujo, R. (2011).Associação entre fissura e perfil antropométrico em dependentes de crack. Jornal Brasileiro de Psiquiatria, 60, 205 - 209.

Drummond, D. (2001). Theories of drug craving, ancient and modern. Addiction, 96, 33- 46.

Elhai, J., Dvorak, R., Levine, J., y Hall, B. (2017). Problematic smartphone use: A conceptual overview and systematic review of relations with anxiety and depression psychopathology. Journal of affective disorders, 207, $251-259$.

Foster, D., Ecker, A., Zvolensky, M., y Buckner, J. (2015). Social anxiety and cannabis cravings: the influences of parent injunctive norms and tension reduction expectancies. Journal of Social and Clinical Psychology, 34, 731 - 746.

Guardia, J., Estorch, M., Surkov, S., del Valle, M., y García, G. (2011). La Escala Multidimensional de Craving de Alcohol y el SPECT con yodobenzamida[I123] como predictores de recaída precoz en pacientes que presentan dependencia del alcohol. Adicciones, 23, 157 - 164.

Guardia, J., Luquero, E., Siñol, N., Burguete, T., y Cardús, M. (2006). Utilidad de la Escala Multidimensional de Craving de Alcohol (EMCA) en la práctica clínica. Adicciones, 18, $265-273$.

Gutierrez, R., Medina-Mora, M., Jiménez, A., Casanova, L., y Natera, G. (2015). Estilos de afrontamiento a la "obsesión mental por beber" (craving) en bebedores en proceso de recuperación. Salud mental, 38, 177 - 183.

Hormes, J., Kearns, B., y Timko, A. (2014). Craving Facebook? Behavioral addiction to online social networking and its association with emotion regulation deficits. Addiction, 109, $2079-2088$.

Ismael, F., y Baltieri, D. (2014). Role of personality traits in cocaine craving throughout an outpatient psychosocial treatment program. Revista Brasileira de Psiquiatria, 36, $24-31$.

Jáuregui, I., Bolaños, P., Carbonero, R., y Valero, E. (2010). Psychometric properties of the Spanish version of Food Craving Inventory (FCI-SP). Nutrición Hospitalaria, 25, 984 - 992.

Jáuregui, I., Bolaños, P., Valero, E., y Ruiz, I. (2012). Introduction to food craving experience; the role of mental imagery, dietary restraint, mood and coping strategies. Nutrición hospitalaria, 27, 1928 - 1935.

Lawrence, S., Luty, J., Bogdan, N., Sahakian, B., y Clark, L. (2009). Problem gamblers share deficits in impulsive decision-making with alcohol-dependent individuals. Addiction, 104, 1006 - 10015.

López, A., y Becoña, E. (2006). El craving en personas dependientes de la cocaína. Anales de psicología, 22, 205 - 211.

Lourido, M. (2015). Elaboración de una escala para evaluar el "Craving" en jugadores patológicos. (Tesis doctoral). Universidad de Barcelona, España.
Magee, J., Lewis, D., y Winhusen, T. (2016). Evaluating Nicotine Craving, Withdrawal, and Substance Use as Mediators of Smoking Cessation in Cocaine - and Methamphetamine - Dependent Patients. Nicotine y Tobacco Research, 18, 1196 - 1201.

Marín, R., Mejía, D., Templos, L., Rosendo, A., González, M., Nanni, R., García, I., Leff, P., Salazar, A., y Antón, B. (2011). Validation of a cocaine craving questionnaire (CCQ-G) in Mexican population. Salud Mental, 34, 491 - 496.

Martin, P., y Petry, N. (2005). Are Non-substance-related Addictions Really Addictions? The American Journal on Addictions, 14, 1 - 7.

Martínez, J., Graña, J., y Trujillo, H. (2010). La calidad de vida en pacientes con trastorno por dependencia al alcohol con trastornos de la personalidad: relación con el ajuste psicológico y craving. Psicothema, 22, 562 - 567.

Martínez, J., Graña, J., y Trujillo, H. (2011). Estudio longitudinal sobre calidad de vida, craving y ajuste psicológico en pacientes dependientes del alcohol: variaciones en función de los trastornos de la personalidad. Adicciones, 23, 227 - 235.

Martínez, J., y Verdejo, A. (2011). Creencias básicas adictivas y craving. Adicciones, 23, 145 - 52.

Merikle, E. (1999). The subjective experience of craving: an exploratory analysis. Substance Use y Misuse, 34, 1101-1115

Montes, V., Urosa, B., Rubio, G., y Poyo, F. (2006). Validación de la escala sobre el deseo de Beber-EDB. Clínica y salud, 17, 203 - 223.

Pedroso, R., Castro, M., y Araujo, R. (2009). Marijuana Craving Questionnaire (MCQ-SF/Versão Brasil): validação semántica. Jornal Brasileiro de Psiquiatria, 58, 218 - 222.

Pérez, L., Quiroga, H., y Pérez, A. (2014). Supresión de pensamientos y días sin consumo de droga comovariables predictivas del craving. Revista Latinoamericana de Medicina Conductual, 4, 83-92.

Piñeiro, B., López, A., Fernández, E., Martínez, U., Brandon, T., y Becoña, E. (2014). Craving and nicotine withdrawal in a Spanish smoking cessation simple. Adicciones, 26, 230 - 237.

Popham, W. (1997). Consequential validity: Right concern - wrong concept. Educational Measurement: Issues and Practice, 16, 9-13.

Skinner, M., y Aubin, H. (2010). Craving's place in addiction theory: Contributions of the major models. Neurosciencie and biobehavioral reviews, 34, $606-623$.

Tiffany, S. (1990). A cognitive model of drug urges and drug-use behavior: Role of automatic and nonautomatic processes. Psychological Review, 97, 147-168.

Torrente, E., Piqueras, J., Orgilés, M., y Espada, J. (2014). Asociación de la adicción a Internet con la ansiedad social y la falta de habilidades sociales en adolescentes españoles. Terapia Psicológica, 32, 175 - 184.

Villalobos, L., Marín, R., Templos, L., y Rosendo, A. (2012). Nicotine craving questionnaire $(\mathrm{CCN})$ : psychometric properties on Mexican population. Salud Mental, 35, 273 - 277. 
Widyanto, L., y Griffiths, M. (2006). Internet addiction: A critical review. International Journal of mental health and Addiction, 4, 31 - 51 .

Zeni, T., y Araujo, R. (2009). O relaxamento respiratório no manejo do craving e dos sintomas de ansiedade em dependentes de crack. Revista de Psiquiatria do Rio Grande do Sul, 31, 116 - 119.

Zeni, T., y Araujo, R. (2011). Relação entre o craving por tabaco e o craving por crack em pacientes internados para desintoxicação. Journal Brasileiro de Psiquiatria, 60, 28 - 33. 